\title{
Management of Spot Blotch (Bipolaris sorokiniana) of Wheat Using Systemic Fungicides
}

\author{
Ghanshyam Verma*, Shivam Kumar, Neeraj Kumar Rajvansi and Santosh Kumar \\ Department of Plant Pathology, N. D. University Kumarganj, Faizabad (U.P.), India \\ *Corresponding author
}

\section{A B S T R A C T}

\begin{tabular}{|l|}
\hline K e y w o r d s \\
Spot blotch, Wheat, \\
Systemic \\
fungicides, Seed \\
treatment
\end{tabular}

\section{Keywords}

Spot blotch, Wheat, Systemic

fungicides, Seed

treatment

Article Info

Available Online:

\section{Introduction}

Spot blotch (Bipolaris sorokiniana (Sacc.) Shoem. of wheat is basically important in North-eastern region, but now it has emerged as serious threat to wheat production in Northwestern region of India along with the Tarai region of North West Plain Zone. Spot blotch of wheat caused by Bipolaris sorokiniana (Sacc.) Shoem has been a major disease of wheat grown under humid subtropical climate (Duveiller, 2002; Roshyara et al., 2009). The disease has a special significance in eastern Gangetic plains of South Asia that includes India, Nepal and
Bangladesh (Sharma and Duveiller, 2004; Joshi et al., 2007). The average yield losses due to spot blotch in India were reported to be 17 percent (Saari, 1998). Therefore, concerted efforts are needed to intensify the research on enhancing the productivity in terms of per unit area on ecologically and economically sustained basis. In this regard due emphasis needs to be given on management of both biotic and abiotic stresses which cause severe loss. Repeated and indiscriminate use of same fungicides often leads to development of fungicide resistance in pathogen (Gangawane, 1997). The variety which is resistant today becomes susceptible in course of time due to 
development of new physiological races of the same pathogen. Therefore advocating suitable fungicides and the manner in which it has to be used by the farmer and at which stage its application, gives maximum benefit was the objective behind carrying this experimentation.

\section{Materials and Methods}

A field study was conducted for two consecutive years (2014-15 and 2015-16), during rabi season at N.D University kumarganj faizabad (u.p.) under natural field condition. The variety Raj 4015 which is highly susceptible to spot blotch was used for the study in both the years. Ten treatments of fungicides with one check were laid out in randomized block design (RBD) with three replications. The plot size was maintained at 1.25 x 2.5 sq.m. and recommended agronomic practices were followed to raise the crop. Four fungicides namely Vitavax 50\% WP, Propiconazole $25 \%$ EC, Tebuconazole $25 \%$ EC and flusilazole $40 \% \mathrm{EC}$ and another one fungicides mixture Propiconazole $13.9 \%+$ difenconazole $13.95 \%$ were applied in the field in different mode with a different spraying schedule. The ten different treatments were, $\mathrm{T} 1=$ Seed Treatment with vitavax power, $\mathrm{T} 2=$ Seed treatment with Vitavax Power @ 2.5g/kg of seed + one foliar spray of Tilt @ $0.1 \%$ at boot leaf or at the time of initiation of disease on flag -1 leaf,T3 = Seed treatment with Vitavax Power@ $2.5 \mathrm{~g} / \mathrm{kg}$ of seed+ one foliar spray of Tilt @ $0.1 \%$ at boot leaf or at the time of initiation of disease on flag -1 leaf fallowed by second spray at 20 days interval (two sprays), $\mathrm{T} 4=$ Seed treatment with Vitavax Power @ 2.5g/ $/ \mathrm{kg}$ of seed +One foliar sprays of Folicur @ 0.1\% at boot leaf or at the time of initiation of disease in flag -1 leaf, T5 = Seed treatment with Vitavax Power @ 2.5g/kg of seed +One foliar sprays of Folicur @ $0.1 \%$ at boot leaf or at the time of initiation of disease in flag -1 leaf fallowed by second spray at 20 days interval (two sprays), T6= Seed treatment with Vitavax Power@2.5g/kg of seed + one foliar spray of Flusilazole@0.1\% at boot leaf or at the time of initiation of disease on flag -1 leaf, T7 $=$ Seed treatment with Vitavax Power @ $2.5 \mathrm{~g} / \mathrm{kg}$ of seed +One foliar sprays of Flusilazole@0.1\% at boot leaf or at the time of initiation of disease in flag -1 leaf fallowed by second spray at 20 days interval (two sprays), T8= Seed treatment with Vitavax Power@2.5g/kg of seed + one foliar spray of Taspa@0.1\% at boot leaf or at the time of initiation of disease on flag -1 leaf, T9= Seed treatment with Vitavax Power @ 2.5g/kg of seed +One foliar sprays of T aspa @ 0.1\% at boot leaf or at the time of initiation of disease in flag -1 leaf fallowed by second spray at 20 days interval (two sprays), T10 $=$ untreated control. The disease data was recorded in three stages (flowering, dough and hard dough) from randomly selected 25 plants from each plot tagged. So, 25 plants plot-1 were tagged for disease rating using the double digit scale (00-99) developed (Eyel et al., 1987).

\section{Results and Discussion}

The results showed that all the treatments reduced the disease severity as evident from the double digit score as well as increased the yield (seed weight /plot)) and yield parameters like 1000 grain weight $(\mathrm{g})$ in comparison to untreated control. The two years data of all the parameters showed differential reaction significantly may be due to different environmental conditions. So, all the recorded parameters of two years data have been presented separately. The results showed that all the fungicides applied plots reduced the disease incidence as well as severity significantly in comparison to untreated control irrespective of their mode of applications. Perusal of the Table 1 and 2 indicates that all the treatments were significantly superior over check (untreated plot) in reducing the disease severity. 
Minimum percent disease intensity in 2014-15 $(12.18 \%)$ in $2015-16$ (11.58\%) was recorded with the treatment $\mathrm{T}_{3}$ (seed treatment with Vitavax Power @ $2.5 \mathrm{~g}$ per kg of seed + foliar spray of Tilt @0.1 per cent at boot leaf or at the time of initiation of disease on flag-1 leaf followed by second spray at 20 days interval) followed by treatment $\mathrm{T}_{5}$ (one foliar spray of Folicur@0.1 per cent at boot leaf or at the time of initiation of disease in flag-1 leaf followed by second spray at 20 days interval) and $\mathrm{T}_{9}$ (one foliar spray of Taspa @ 0.1 per cent at boot leaf or at the time of initiation of disease on flag-1 leaf followed by second spray at 20 days interval) which have disease intensity 2014-15 of 23.35 percent and 45.32 per cent and in 2015-16 22.89\% and $44.70 \%$ respectively. Former treatment was significantly superior over the latter. All the other treatments also have maximum percent disease intensity over check, significantly except treatment $\mathrm{T}_{1}, \mathrm{~T}_{2} \mathrm{~T} 4, \mathrm{~T} 6$, and $\mathrm{T} 8$.

Table.1 Evaluation of different fungicides against foliar blight (2014-15)

\begin{tabular}{|c|l|l|l|l|l|l|l|l|l|}
\hline Treatments & $\begin{array}{l}\text { Date of } \\
\text { Disease } \\
\text { appearance }\end{array}$ & $\begin{array}{l}\text { Before } \\
\text { spraying }\end{array}$ & $\begin{array}{l}\text { After } \\
\text { first } \\
\text { spray }\end{array}$ & $\begin{array}{l}\text { After } \\
\text { second } \\
\text { spray }\end{array}$ & PDI & AUDPC & $\begin{array}{l}\text { Yield } \\
\text { Kg/ha }\end{array}$ & $\begin{array}{l}\text { Yield } \\
\text { q/ha }\end{array}$ & $\begin{array}{l}1000 \\
\text { grain } \\
\text { wt. }\end{array}$ \\
\hline $\mathbf{T}_{\mathbf{1}}$ & $26 / 1 / 15$ & 15.33 & $\begin{array}{l}42.23 \\
(40.51)\end{array}$ & $\begin{array}{l}52.12 \\
(46.26)\end{array}$ & $\begin{array}{l}67.26 \\
(55.06)\end{array}$ & 629.6 & 1.038 & 33.20 & 39.48 \\
\hline $\mathbf{T}_{\mathbf{2}}$ & $24 / 1 / 15$ & 0.75 & $\begin{array}{l}23.67 \\
(29.06)\end{array}$ & $\begin{array}{l}28.18 \\
(32.01)\end{array}$ & $\begin{array}{l}35.25 \\
(36.39)\end{array}$ & 348.3 & 1.195 & 38.24 & 40.19 \\
\hline $\mathbf{T}_{\mathbf{3}}$ & $26 / 1 / 15$ & 0.00 & $\begin{array}{l}4.33 \\
(11.92)\end{array}$ & $\begin{array}{l}7.44 \\
(15.70)\end{array}$ & $\begin{array}{l}12.18 \\
(20.36)\end{array}$ & 111.5 & 1.201 & 38.44 & 41.52 \\
\hline $\mathbf{T}_{\mathbf{4}}$ & $26 / 1 / 15$ & 0.85 & $\begin{array}{l}25.12 \\
(30.07)\end{array}$ & $\begin{array}{l}27.13 \\
(31.37)\end{array}$ & $\begin{array}{l}46.25 \\
(42.82)\end{array}$ & 435.3 & 1.160 & 37.12 & 39.36 \\
\hline $\mathbf{T}_{\mathbf{5}}$ & $22 / 1 / 15$ & 0.15 & $\begin{array}{l}12.45 \\
(20.62)\end{array}$ & $\begin{array}{l}18.33 \\
(25.33)\end{array}$ & $\begin{array}{l}23.35 \\
(28.86)\end{array}$ & 196.9 & 1.173 & 37.52 & 40.69 \\
\hline $\mathbf{T}_{\mathbf{6}}$ & $23 / 1 / 15$ & 1.00 & $\begin{array}{l}27.36 \\
(30.50)\end{array}$ & $\begin{array}{l}30.55 \\
(33.46)\end{array}$ & $\begin{array}{l}57.22 \\
(49.14)\end{array}$ & 516.5 & 1.075 & 34.40 & 38.29 \\
\hline $\mathbf{T}_{\mathbf{7}}$ & $25 / 1 / 15$ & 0.85 & $\begin{array}{l}26.55 \\
(30.98)\end{array}$ & $\begin{array}{l}33.34 \\
(35.24)\end{array}$ & $\begin{array}{l}47.23 \\
(43.39)\end{array}$ & 443.4 & 1.091 & 34.92 & 38.40 \\
\hline $\mathbf{T}_{\mathbf{8}}$ & $26 / 1 / 15$ & 1.00 & $\begin{array}{l}32.26 \\
(34.52)\end{array}$ & $\begin{array}{l}39.60 \\
(39.00)\end{array}$ & $\begin{array}{l}56.46 \\
(48.68)\end{array}$ & 505.1 & 1.143 & 36.56 & 39.16 \\
\hline $\mathbf{T}_{\mathbf{9}}$ & $23 / 1 / 15$ & 1.00 & $\begin{array}{l}30.56 \\
(33.52)\end{array}$ & $\begin{array}{l}34.30 \\
(35.85)\end{array}$ & $\begin{array}{l}45.32 \\
(42.80)\end{array}$ & 428.2 & 1.148 & 36.72 & 39.67 \\
\hline $\mathbf{T}_{\mathbf{1 0}}$ & $26 / 1 / 15$ & 32.54 & $\begin{array}{l}44.67 \\
(41.90)\end{array}$ & $\begin{array}{l}67.88 \\
(55.43)\end{array}$ & $\begin{array}{l}79.36 \\
(62.53)\end{array}$ & 771.9 & 0.890 & 28.48 & 29.01 \\
\hline SEm \pm & & 0.24 & 3.27 & 2.72 & $\mathbf{0 . 4 6 4}$ & & $\mathbf{0 . 0 4}$ & $\mathbf{1 . 1 2}$ & 1.99 \\
\hline CD 5 \% & & 0.76 & 9.56 & 7.92 & $\mathbf{1 . 3 4 7}$ & & $\mathbf{0 . 1 0}$ & $\mathbf{3 . 2 5}$ & 5.79 \\
\hline
\end{tabular}


Table.2 Evaluation of different fungicides against foliar blight (2015-16)

\begin{tabular}{|c|c|c|c|c|c|c|c|c|c|}
\hline $\begin{array}{l}\text { No. of } \\
\text { treatment }\end{array}$ & $\begin{array}{l}\text { Date of } \\
\text { Disease } \\
\text { appearance }\end{array}$ & $\begin{array}{l}\text { Before } \\
\text { spraying }\end{array}$ & $\begin{array}{l}\text { After } \\
\text { first } \\
\text { spray }\end{array}$ & $\begin{array}{l}\text { After } \\
\text { second } \\
\text { spray }\end{array}$ & PDI & AUDPC & $\begin{array}{l}\text { Yield } \\
\mathrm{Kg} / \mathrm{ha}\end{array}$ & $\begin{array}{l}\text { Yield } \\
\text { q/ha }\end{array}$ & $\begin{array}{l}1000 \\
\text { grain } \\
\text { wt. }\end{array}$ \\
\hline $\mathrm{T}_{1 \text { STVP }}$ & 28/1/15 & $\begin{array}{l}14.45 \\
(22.30)\end{array}$ & $\begin{array}{l}39.56 \\
(38.94)\end{array}$ & $\begin{array}{l}49.66 \\
(44.77)\end{array}$ & $\begin{array}{l}66.85 \\
(54.82)\end{array}$ & 623.0 & 1.078 & 34.48 & 38.80 \\
\hline $\begin{array}{l}\mathrm{T}_{2 \text { STVP }+1} \\
\text { tilt spray }\end{array}$ & 27/1/15 & $\begin{array}{l}0.64 \\
(4.59)\end{array}$ & $\begin{array}{l}21.43 \\
(27.53)\end{array}$ & $\begin{array}{l}26.76 \\
(31.11)\end{array}$ & $\begin{array}{l}34.39 \\
(35.85)\end{array}$ & 337.8 & 1.223 & 39.12 & 41.15 \\
\hline $\begin{array}{l}\mathrm{T}_{3 \text { STVP }+2} \\
\text { tilt spray }\end{array}$ & 27/1/15 & 0.00 & $\begin{array}{l}3.76 \\
(11.09)\end{array}$ & $\begin{array}{l}6.89 \\
(15.12)\end{array}$ & $\begin{array}{l}11.58 \\
(19.82)\end{array}$ & 107.4 & 1.233 & 39.44 & 42.17 \\
\hline $\begin{array}{l}\mathrm{T}_{4} \text { STVP }+1 \\
\text { folicur spray }\end{array}$ & 29/1/15 & $\begin{array}{l}0.83 \\
(5.23)\end{array}$ & $\begin{array}{l}23.65 \\
(29.06)\end{array}$ & $\begin{array}{l}25.29 \\
(30.13)\end{array}$ & $\begin{array}{l}45.83 \\
(42.59) \\
\end{array}$ & 426.2 & 1.198 & 38.32 & 39.94 \\
\hline $\begin{array}{l}\mathrm{T}_{5 \text { STVP }+2} \\
\text { folicur spray }\end{array}$ & 27/1/15 & $\begin{array}{l}0.13 \\
(2.07)\end{array}$ & $\begin{array}{l}11.43 \\
(19.73)\end{array}$ & $\begin{array}{l}17.34 \\
(24.58)\end{array}$ & $\begin{array}{l}22.89 \\
(28.52)\end{array}$ & 191.6 & 1.200 & 38.40 & 41.76 \\
\hline $\begin{array}{l}\mathrm{T}_{6} \quad \text { STVP } \\
+1 \text { Flusilazole } \\
\text { spray }\end{array}$ & 28/1/15 & $\begin{array}{l}0.96 \\
(5.62)\end{array}$ & $\begin{array}{l}25.87 \\
(30.53)\end{array}$ & $\begin{array}{l}28.62 \\
(32.33)\end{array}$ & $\begin{array}{l}56.84 \\
(48.94)\end{array}$ & 509.7 & 1.105 & 35.36 & 38.45 \\
\hline $\begin{array}{l}\mathrm{T}_{7} \quad \text { STVP } \\
+2 \text { Flusilazole } \\
\text { spray }\end{array}$ & 28/1/15 & $\begin{array}{l}0.83 \\
(5.23)\end{array}$ & $\begin{array}{l}24.33 \\
(29.53)\end{array}$ & $\begin{array}{l}31.33 \\
(34.02)\end{array}$ & $\begin{array}{l}46.65 \\
(43.05)\end{array}$ & 433.6 & 1.113 & 35.60 & 39.05 \\
\hline $\begin{array}{l}\mathrm{T}_{8 \mathrm{STVP}+1} \\
\text { taspa spray }\end{array}$ & 29/1/15 & $\begin{array}{l}0.97 \\
(5.65)\end{array}$ & $\begin{array}{l}31.68 \\
(34.20)\end{array}$ & $\begin{array}{l}37.46 \\
(37.70)\end{array}$ & $\begin{array}{l}55.81 \\
(48.33)\end{array}$ & 495.9 & 1.160 & 37.12 & 38.13 \\
\hline $\begin{array}{l}\mathrm{T}_{9} \mathrm{STVP}+2 \\
\text { taspa spray }\end{array}$ & 27/1/15 & $\begin{array}{l}0.93 \\
(5.53)\end{array}$ & $\begin{array}{l}29.88 \\
(33.09)\end{array}$ & $\begin{array}{l}32.89 \\
(34.94)\end{array}$ & $\begin{array}{l}44.70 \\
(41.96)\end{array}$ & 422.1 & 1.173 & 37.52 & 41.19 \\
\hline $\mathrm{T}_{10 \text { Control }}$ & 24/1/15 & $\begin{array}{l}0.98 \\
(5.68)\end{array}$ & $\begin{array}{l}41.72 \\
(40.22)\end{array}$ & $\begin{array}{l}65.89 \\
()\end{array}$ & $\begin{array}{l}78.61 \\
(62.44)\end{array}$ & 760.6 & 0.940 & 30.08 & 30.98 \\
\hline SEm \pm & & 0.24 & 2.56 & 3.21 & 0.51 & & 0.02 & 0.78 & 1.73 \\
\hline CD $5 \%$ & & 0.76 & 5.39 & 8.73 & 1.48 & & 0.07 & 2.27 & 5.01 \\
\hline
\end{tabular}

\section{Grain yield}

In case of seed yield similar trend was followed, in 2014-15 maximum being with treatment $\mathrm{T}_{3}(1.201 \mathrm{~kg} / \mathrm{plot}$ and $38.448 \mathrm{q}$ per ha.) and $\mathrm{T}_{5}(1.173 \mathrm{~kg} / \mathrm{plot}$ and $37.52 \mathrm{q}$ per ha.) and in $2015-16 \mathrm{~T}_{3}(1.233 \mathrm{~kg} / \mathrm{plot}$ and $39.44 \mathrm{q}$ per ha.) and $\mathrm{T}_{5}(1.200 \mathrm{~kg} / \mathrm{plot}$ and $38.40 \mathrm{q}$ per ha.) respectively, but all were at par. Again similar trend was followed with thousand grain weight. All the other treatments also increased the seed yield over check, significantly except treatment $\mathrm{T}_{1}, \mathrm{~T}_{2} \mathrm{~T} 4$, T6, and $\mathrm{T} 8$

\section{Thousand grain weight (g)}

Yield attribute like 1000 grain weight (g) also showed the same trends as observed in grain yield. Both the years (2014-15 and 2015-16) maximum 1000 grain weight was observed in T3 (41.52 and 42.17 respectively) followed 
by T5 (40.69 and 41.76 respectively) (Table 1 and 2).

Percent disease intensity was calculated by employing the formula:

P.D. $\mathrm{I}=\frac{\text { Sum of total numerical rating }}{\text { Total number of leaves examined } \mathrm{x} \text { Highest rating }} \times 100$

\section{AUDPC}

$\sum_{i}^{n-1}\left[\left(\frac{y_{i}+y_{i+1}}{2}\right)\left(t_{i+1}-t_{i}\right)\right]$

\section{References}

Duveiller E (2002). Helminthosporium leaf blight of wheat challenges and strategies for a better disease control. In: Advances of wheat breeding in China. Proceedings of the first National Wheat Breeding Conference. 10-12 May, 2000, pp. 57- 66.

Eyal Z, Scharen AL, Prescott JM and Van Ginkel M (1987). The Septoria disease of wheat: concepts and methods of disease management. CIMMYT. Mexico. D.F.

Gangawane LV (1997). Management of fungicide resistance in Plant Pathogen. Indian Phytopathology 50: 305-313.

Joshi AK, Ortiz Ferrara G, Crossa J, Singh G,
Sharma R, Chand R and Prasad R (2007). Combining superior agronomic performance and terminal heat tolerance with resistance to spot blotch (Bipolaris sorokiniana) in warm humid Gangetic plains of South Asia. Field Crop Research 103:53-61.

Mahpatra, Sunita and Das Saikat (2013) Efficcay of different fungicides against spot blotch of wheat in terai regions of West Bengal. J.Wheat.Res. 5(2):18-21.

Roshyara UR, Khadka K, Subedi S, Sharma RC and Duveiller E (2009). Field resistance to spot blotch is not associated with underside physiomorphological traits in three wheat spring population. Journal of Plant Pathology, 91(1): 113-122.

Saari EE (1998). Leaf blight diseases and associate soil borne fungus pathogens of Wheat in South and Southeast Asia. In: Duveiller E., Dubin N.J., Reeves J. and $\mathrm{McNab}$ A. (Eds.): Helminthosporium blight of wheat: spot blotch and Tan spot CYMMYT. Mexico, D.F.: 37-51.

Sharma RC and Duveiller E (2004). Effect of Helminthosporium leaf blight on performance of timely and late seeded wheat under optimal and stressed levels of soil fertility and moisture. Field Crops Research 89: 205-218.

\section{How to cite this article:}

Ghanshyam Verma, Shivam Kumar, Neeraj Kumar Rajvansi and Santosh Kumar. 2018. Management of Spot Blotch (Bipolaris sorokiniana) of Wheat Using Systemic Fungicides. Int.J.Curr.Microbiol.App.Sci. 7(08): 4277-4281. doi: https://doi.org/10.20546/ijcmas.2018.708.448 